\title{
The effect of sedation with a combination of butorphanol and midazolam on quantitative contrast-enhanced ultrasonography of duodenum in healthy dogs
}

\author{
Khoirun NISA ${ }^{1)}$, Sue Yee LIM ${ }^{1,5)}$, Tatsuyuki OSUGA ${ }^{1)}$, Nozomu YOKOYAMA ${ }^{1,4}$, \\ Masahiro TAMURA ${ }^{1)}$, Noriyuki NAGATA ${ }^{1)}$, Kazuyoshi SASAOKA ${ }^{1)}$, \\ Angkhana DERMLIM ${ }^{1)}$, Rommaneeya LEELA-ARPORN ${ }^{1)}$, Tomoya MORITA ${ }^{1)}$, \\ Noboru SASAKI ${ }^{1}$, Keitaro MORISHITA ${ }^{2)}$, Kensuke NAKAMURA ${ }^{2,3)}$, Hiroshi OHTA ${ }^{1)}$ \\ and Mitsuyoshi TAKIGUCHI ${ }^{1) *}$
1)Laboratory of Veterinary Internal Medicine, Department of Veterinary Clinical Sciences, Graduate School of Veterinary Medicine, Hokkaido University, Sapporo, Hokkaido 060-0818, Japan
${ }^{2)}$ Veterinary Teaching Hospital, Graduate School of Veterinary Medicine, Hokkaido University, Sapporo, Hokkaido 060-0818, Japan
3) Organization for promotion of Tenure Track, University of Miyazaki, Miyazaki 889-2192, Japan
${ }^{4)}$ Department of Veterinary Internal Medicine, Graduate School of Agricultural and Life Sciences, The University of Tokyo, Tokyo 113-8657, Japan
${ }^{5)}$ Department of Veterinary Clinical Sciences, Faculty of Veterinary Medicine, Universiti Putra Malaysia, Serdang, \\ Selangor 43400, Malaysia
}

J. Vet. Med. Sci.

80(3): 453-459, 2018

doi: 10.1292/jvms.17-0525

Received: 25 September 2017 Accepted: 22 January 2018 Published online in J-STAGE: 2 February 2018

\begin{abstract}
Quantitative contrast-enhanced ultrasonography (CEUS) enables non-invasive and objective evaluation of intestinal perfusion by quantifying the intensity of enhancement on the intestine after microbubble contrast administration. During CEUS scanning, sedation is sometimes necessary to maintain animal cooperation. Nevertheless, the effect of sedative administration on the canine intestinal CEUS is unknown. This study aimed to investigate the effect of sedation with a combination of butorphanol and midazolam on the duodenal CEUSderived perfusion parameters of healthy dogs. For this purpose, duodenum was imaged following contrast administration (Sonazoid ${ }^{\circ}, 0.01 \mathrm{~m} / / \mathrm{kg}$ ) in six healthy beagles before and after intravenous injection of a combination of butorphanol $(0.2 \mathrm{mg} / \mathrm{kg})$ and midazolam $(0.1 \mathrm{mg} / \mathrm{kg})$. Furthermore, hemodynamic parameters including blood pressure and heart rate were recorded during the procedure. Five CEUS derived perfusion parameters including time-to-peak (TTP), peak intensity $(\mathrm{PI})$, area under the curve (AUC), wash-in and wash-out rates (WiR and WoR, respectively) before and after sedation were statistically compared. The result showed that no significant change was detected in any of perfusion parameters. Systolic and mean arterial pressures significantly reduced after sedative administration, but diastolic arterial pressure and heart rate did not significantly change. Moreover, no significant partial correlation was observed between perfusion parameters and hemodynamic parameters. Thus, we concluded that the combination did not cause significant influence in duodenal CEUS perfusion parameters and could be a good option for sedation prior to duodenal CEUS in debilitated dogs.

KEY WORDS: butorphanol-midazolam, canine, duodenal CEUS, sedative effect
\end{abstract}

Quantitative contrast-enhanced ultrasonography (CEUS) enables non-invasive and objective evaluation of tissue perfusion by quantifying the intensity of contrast enhancement on the targeted tissue after microbubble contrast administration. The intensity of enhancement is proportional to the amount of microbubbles that remain strictly intravascular. The quantification is conducted by placing a region of interest (ROI) on the targeted tissue and constructing a time-intensity curve (TIC) which reflects the wash-in and wash-out phase of the contrast agent within ROI. The TIC-derived perfusion parameters depict regional blood flow and blood volume of the analyzed tissue [5].

Quantitative CEUS has been growingly utilized to evaluate various organs both in human and veterinary medicine including

*Correspondence to: Takiguchi, M.: mtaki@vetmed.hokudai.ac.jp

O2018 The Japanese Society of Veterinary Science

This is an open-access article distributed under the terms of the Creative Commons Attribution Non-Commercial No Derivatives (by-nc-nd) License. (CC-BY-NC-ND 4.0: https://creativecommons.org/licenses/by-nc-nd/4.o/) 
liver, spleen, and pancreas [19, 21, 32-34]. In human medicine, the perfusion parameters obtained from a quantitative analysis of intestinal CEUS were reported to allow estimation of disease activity, evaluation of treatment response, as well as differentiation between fibrosis and active inflammation in patients with inflammatory bowel disease [8, 20, 24-26]. A few studies on the characterization of intestinal perfusion using quantitative CEUS in healthy dogs have been reported [10, 11, 19]. In addition, the preliminary study of duodenal CEUS in our institution indicated that the repeatability and reproducibility of this modality were clinically acceptable in healthy dogs [22]. Thus, clinical application of this modality to detect changes of intestinal perfusion which is expected to occur related to inflammatory (i.e. idiopathic inflammatory bowel disease) and neoplastic diseases (i.e. intestinal lymphoma) in dogs is subjected in the near future.

In veterinary studies, it is important to maintain animal cooperation during CEUS acquisition because animal movement interferes with the ROI placement. For this purpose, a sedative is sometimes required to restrain debilitated animals. A combination of butorphanol and midazolam is commonly used to produce sedation and analgesia for minimally invasive diagnostic procedure in dogs [18]. This combination was reported to have minimal effect on the cardiopulmonary system [14], thus it is expected to be less likely in interfering with the CEUS interpretation. Moreover, it has a rapid onset and short duration of action that make it an ideal choice for the short-term procedure. Unfortunately, the effect of this combination on the perfusion parameters obtained from canine intestinal CEUS has not been reported. Therefore, the aim of current study was to investigate the effect of sedation with a combination of butorphanol and midazolam on the duodenal CEUS-derived perfusion parameters of healthy dogs.

\section{MATERIALS AND METHODS}

Six beagle dogs (three intact males and three intact females, aged 1-4 years, weighed $8.8-12 \mathrm{~kg}$ ) were studied. The sample size was determined based on a previous report on the sedative effect of CEUS parameters in other organs [28, 31]. All dogs were healthy based on normal physical examination, blood count, and biochemistry (e.g. albumin, C-reactive protein, lipase). No dogs showed clinical signs related to gastrointestinal diseases. Neither focal nor diffuse abnormalities were found in the duodenum of all dogs when B-mode ultrasonography was performed. Dogs fasted for approximately $12 \mathrm{hr}$ before the experiment. All procedures in the current study were approved by the Hokkaido University Animal Care and Use Committee (Approval no. 16-0094).

Each dog underwent CEUS twice on the same day with at least 1-hr wash-out interval. Baseline CEUS was performed before sedation, whereas post-sedation CEUS was performed at approximately 15-30 min after sedative administration. The sedative drugs used in this study composed by butorphanol (Vetorphale ${ }^{\circledR} 5 \mathrm{mg} / \mathrm{ml}$, Meiji Seika Pharma Co., Ltd., Tokyo, Japan) and midazolam (Dormicum ${ }^{\circledR} 5 \mathrm{mg} / \mathrm{ml}$, Astellas Pharma Inc., Tokyo, Japan). The mixture was administered intravenously in a dosage of 0.2 and $0.1 \mathrm{mg} / \mathrm{kg}$ for butorphanol and midazolam, respectively. The sedative effect was confirmed by a decrease in blood pressure, lower jaw tone, and less response to sound and contact $[14,15]$.

CEUS acquisition was undertaken using an ultrasound system (Aplio XG, Toshiba Medical Systems, Otawara, Japan) with a 5-11 MHz linear probe (PLT-704 AT, Toshiba Medical Systems). Mechanical index (MI) was adjusted at 0.20. Focal depth was placed at $2 \mathrm{~cm}$. Gain and dynamic range were set at 75 and $45 \mathrm{~dB}$. These parameters were optimized during the preliminary study and adjusted for all dogs. Dogs were positioned in left lateral recumbency and the probe was placed behind the last rib to image longitudinal view of the duodenum. Contrast agent (Sonazoid $\AA$, Daiichi-Sankyo, Tokyo, Japan) was administered as an intravenous bolus in a dosage of $0.01 \mathrm{ml} / \mathrm{kg}$ and flushed with $2 \mathrm{~m} l$ heparin saline. The image was recorded for a total of $120 \mathrm{sec}$ following contrast administration. All CEUS was performed by one sonographer (K.N.). In addition, hemodynamic parameters including indirect blood pressure [(BP): systolic arterial pressure (SAP), diastolic arterial pressure (DAP), mean arterial pressure (MAP)] and heart rate (HR) were monitored noninvasively during CEUS scanning using a patient monitor with an oscillometric BP device (BSM-5192, Nihon Kohden Co., Tokyo, Japan).

Quantitative analysis of CEUS images was done using image analysis software (ImageJ, US National Institutes of Health, Bethesda, MD, U.S.A.) by one observer (K.N.). One frame per sec for a total $120 \mathrm{sec}$ was analyzed. Four ROIs were manually drawn as large as possible in the duodenal mucosa at approximately the same depth and without including big vessels or adjacent tissue. If four ROIs could not be drawn due to motion artifacts, one, two, or three ROIs were drawn instead. When a respiratory motion or duodenal movement was present, the ROIs were adjusted manually to maintain the same position within the duodenal mucosa and narrowed depth range. The software calculated the intensity within each ROI as a gray-scale level ranging from a mean pixel value (MPV) of 0-255. The intensity data were subsequently exported to a commercial software (Microsoft Excel 2013, Microsoft, Redmond, WA, U.S.A.), followed by averaging the intensities obtained from the four ROIs. The average intensities were plotted against time to create a time-intensity curve (TIC).

A number of perfusion parameters were acquired from TIC, including the time-to-peak (TTP), which refers to the time from the arrival time (AT, time point when the intensity is above the baseline, and followed by a further rise) to maximum enhancement; peak intensity (PI) refers to maximum enhancement with subtraction of the baseline intensity (BI, intensity at AT); area under the curve (AUC) refers to the area under the TIC curve above BI and was calculated from AT to 120 sec; wash-in and wash-out rates (WiR and WoR, respectively) refer to the slope of ascending and descending tracts of TIC respectively. WiR and WoR were calculated by performing regression using the same Excel sheet to subsequent points that continued to increase from BI to PI and decrease from PI to the end of the recording, respectively.

Statistical analysis was done using statistical analysis programs (JMP pro 12.0.1, SAS Institute Inc., Cary, NC, U.S.A. and IBM SPSS Statistics V22.0, IBM Corp., Armonk, NY, U.S.A.). All parameters were evaluated for normal distribution using ShapiroWilk Test, and the results are expressed as the mean \pm standard deviation (SD). Values of all perfusion parameters before and after 

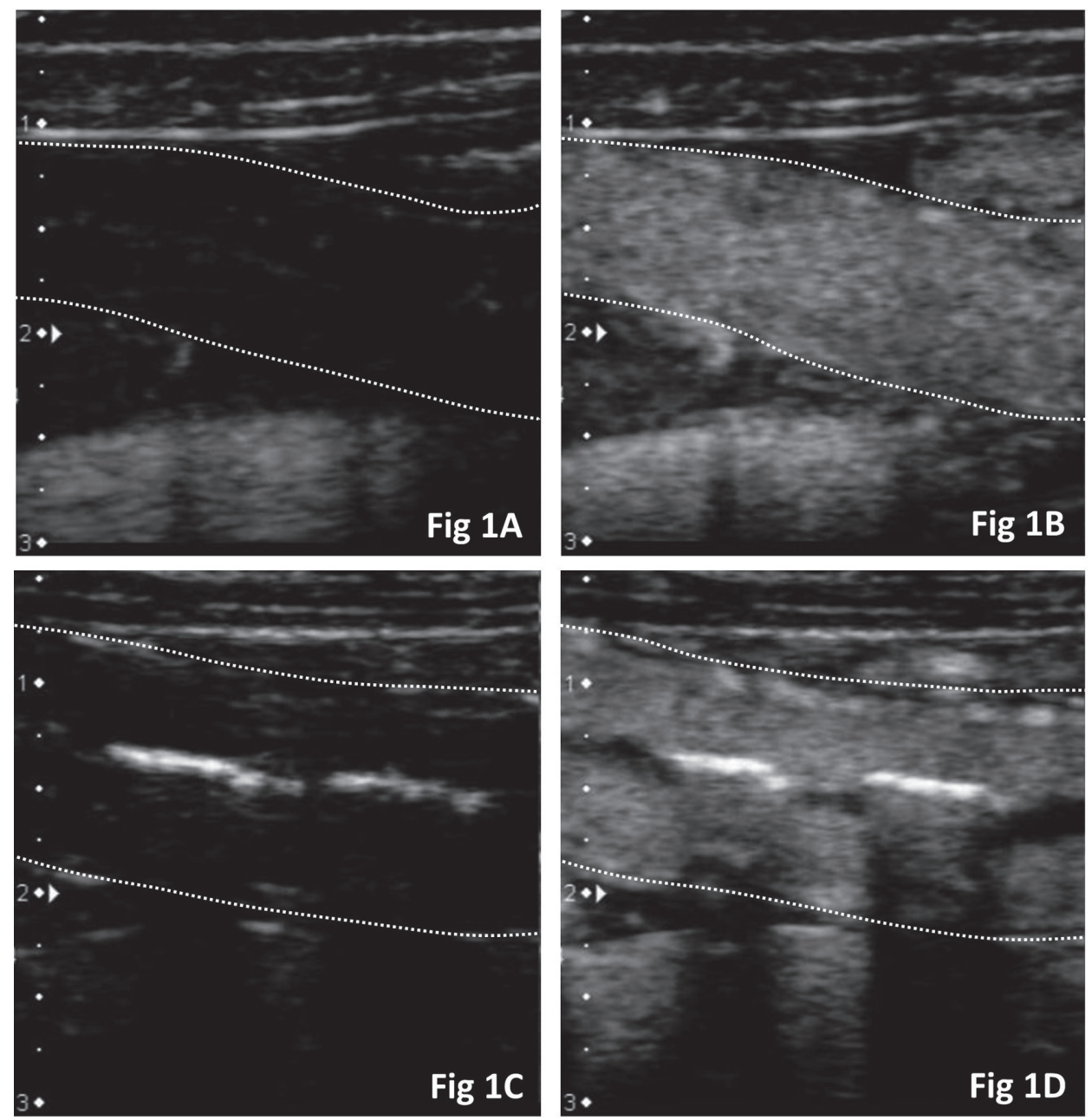

Fig. 1. CEUS images of the duodenum at arrival time and peak enhancement of one representative $\operatorname{dog}$ before $[\mathrm{A}, \mathrm{B}]$ and after sedation $[\mathrm{C}, \mathrm{D}]$. Duodenum (dashed line) shows no enhancement at the arrival time of contrast agent in the dog before $[\mathrm{A}]$ and after [C] sedation. The duodenum is similarly enhanced at peak intensity before $[\mathrm{B}]$ and after [D] sedation.

sedation were compared using paired student's $t$ test for normally distributed values, or using a non-parametric test (Wilcoxon rank-sum test) for nonnormally distributed values. Furthermore, the differences of these parameters before and after sedation were evaluated by using Bland-Altman analysis [2]. Mean differences (bias), 95\% confidence intervals (CIs) for bias, and limits of agreement (LoAs) were calculated. Bias before and after sedation was considered significant when the $95 \%$ CI did not contain 0 . Results of the Bland-Altman analysis are summarized as least square mean $(95 \% \mathrm{CI})$ of bias for each parameter.

The data of SAP, DAP, MAP and HR were analyzed using mixed model for repeated measures with time $(0,5,10,15,20,25$ and $30 \mathrm{~min}$ ) as fixed effect and dog as a random effect. The $F$ test was performed to assess the effect of time on the values of repeated measures. Pairwise comparisons between times were performed by calculating least square means and using Bonferroni correction to adjust the multiple comparisons. In addition, the partial correlation between hemodynamic and perfusion parameters was also analyzed. For partial correlation analysis, the value of SAP, DAP, MAP and HR which was detected at the contrast agent injection were used. Values of $P<0.05$ were considered significant for all analyses.

\section{RESULTS}

All CEUS images obtained from six dogs were adequate for analysis. CEUS images before and after sedation of one representative dog at the arrival time and peak intensity are presented (Fig. 1A-D). The duodenum of this dog is similarly enhanced at peak intensity in the awaken state and after sedative administration (Fig. 1B and 1D, respectively). The TIC derived from duodenal CEUS of the representative dog are presented in Fig. 2. In this representative dog, TIC before and after sedation show no different pattern.

Mean and standard deviation of all perfusion parameters obtained from duodenal CEUS before and after sedation are given in Table 1. Bland Altman analysis confirmed no significant difference in any of perfusion parameters before and after sedation (Fig. $3 \mathrm{~A}-\mathrm{E})$. The SAP is significantly lower at $25 \mathrm{~min}$, while the MAP is significantly lower at 5, 10, 15 and 25 min when compared to 


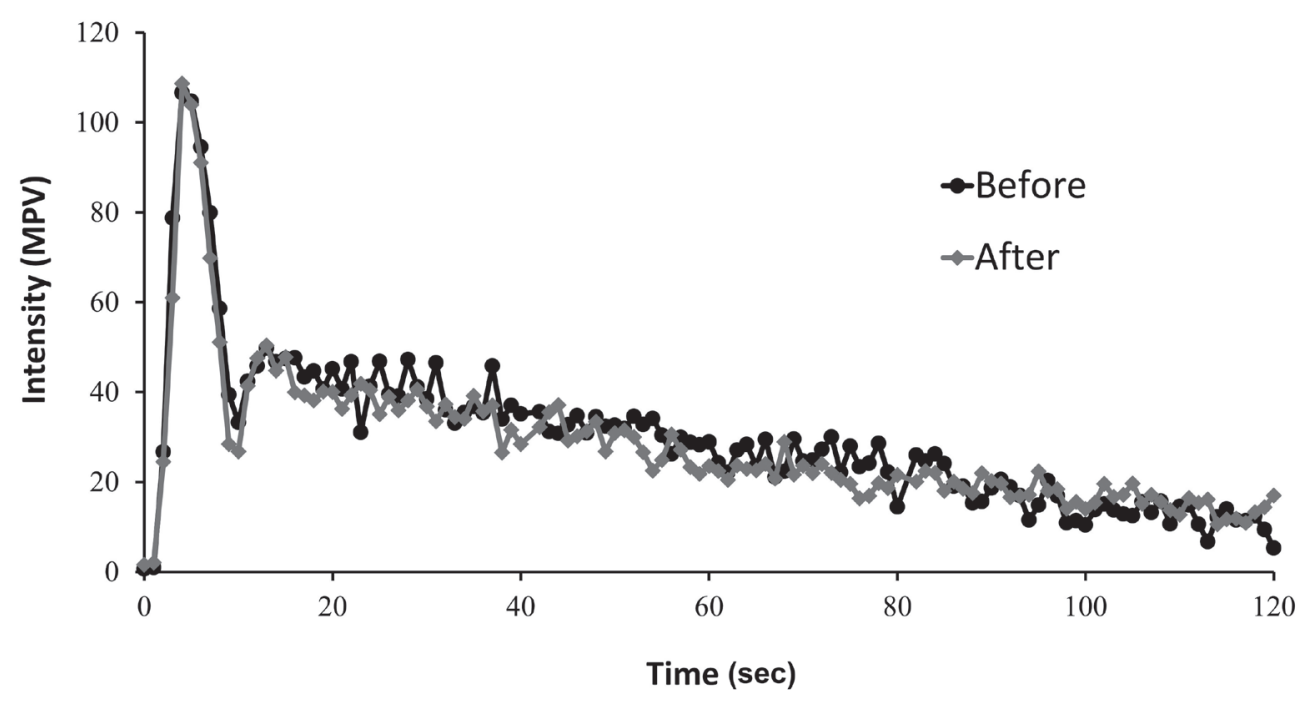

Fig. 2. Time-intensity curve (TIC) derived from duodenal CEUS of one representative dog before and after sedation. In this dog, TIC before and after sedation are similar.

Table 1. Mean and standard deviation of perfusion parameters derived from duodenal CEUS before and after sedation

\begin{tabular}{lcc}
\hline \multicolumn{1}{c}{ Variable } & Before sedation & After sedation \\
\hline Perfusion parameters & & \\
TTP (sec) & $4.67 \pm 1.51$ & $4.33 \pm 1.03$ \\
PI (MPV) & $89.28 \pm 11.07$ & $89.15 \pm 9.88$ \\
AUC (MPV/sec) & $2,536.18 \pm 747.43$ & $2,428.11 \pm 814.14$ \\
WiR (MPV/sec) & $22.89 \pm 8.82$ & $23.32 \pm 6.47$ \\
WoR (MPV/sec) & $-0.72 \pm 0.11$ & $-0.71 \pm 0.06$ \\
\hline
\end{tabular}

Data are expressed as mean $\pm \mathrm{SD}(\mathrm{n}=6)$. TTP, time to peak; WiR, wash in rate; WoR, wash out rate; PI, peak intensity, AUC, area under curve; MPV, mean pixel value.

baseline (time $=0$, before sedative administration) (Fig. 4A). DAP and HR did not show significant difference at any time point (Fig. 4A and $4 B$ ). No significant partial correlation was found between perfusion and hemodynamic parameters.

\section{DISCUSSION}

In the present study, perfusion parameters derived from duodenal CEUS of healthy dogs including TTP, PI, AUC, WiR and WoR were evaluated before and after sedation using a combination of butorphanol and midazolam. No significant change was observed in any of these parameters. This finding indicated that the combination could be a good option for sedation prior to duodenal CEUS in debilitated dogs.

Butorphanol produces an analgesic effect by its action at $\kappa$ and $\mu$ receptors of central nervous system [16], whereas midazolam interacts with gamma-aminobutyric acid (GABA) receptor to produce muscle relaxation [18]. The combination of butorphanol and midazolam was reported to promote light to moderate sedation with small changes in cardiopulmonary functions [14]. Moreover, their rapid onset and short duration of action make them a safe and ideal option for a short-term procedure like CEUS. For this reason, the combination was selected for current study with the expectation that it would have less influence on the duodenal perfusion parameters.

The effect of butorphanol-midazolam on CEUS parameters of the canine duodenum was first examined in the current study. The use of butorphanol as a single sedative agent was suggested to cause neither significant change on the CEUS parameters of canine spleen nor those of feline kidney, that make this agent was selectable to be utilized during CEUS scanning of both organs [28, 31]. Butorphanol-midazolam, however, was reported to provoke a reduction of intrarenal blood flow in healthy dogs by Doppler ultrasound [23]. The latter finding was in contrast to our result, but the direct comparison could not be made. It was not only because duodenum and kidney may respond differently to this combination, but also due to the difference in dosage. The dosage of midazolam used in the study above was twice of the dosage we injected to our dogs. In our study, midazolam was administered in a dosage of $0.1 \mathrm{mg} / \mathrm{kg}$ which was reported to produce adequate sedation in healthy beagles when combined with $0.2 \mathrm{mg} / \mathrm{kg}$ of 

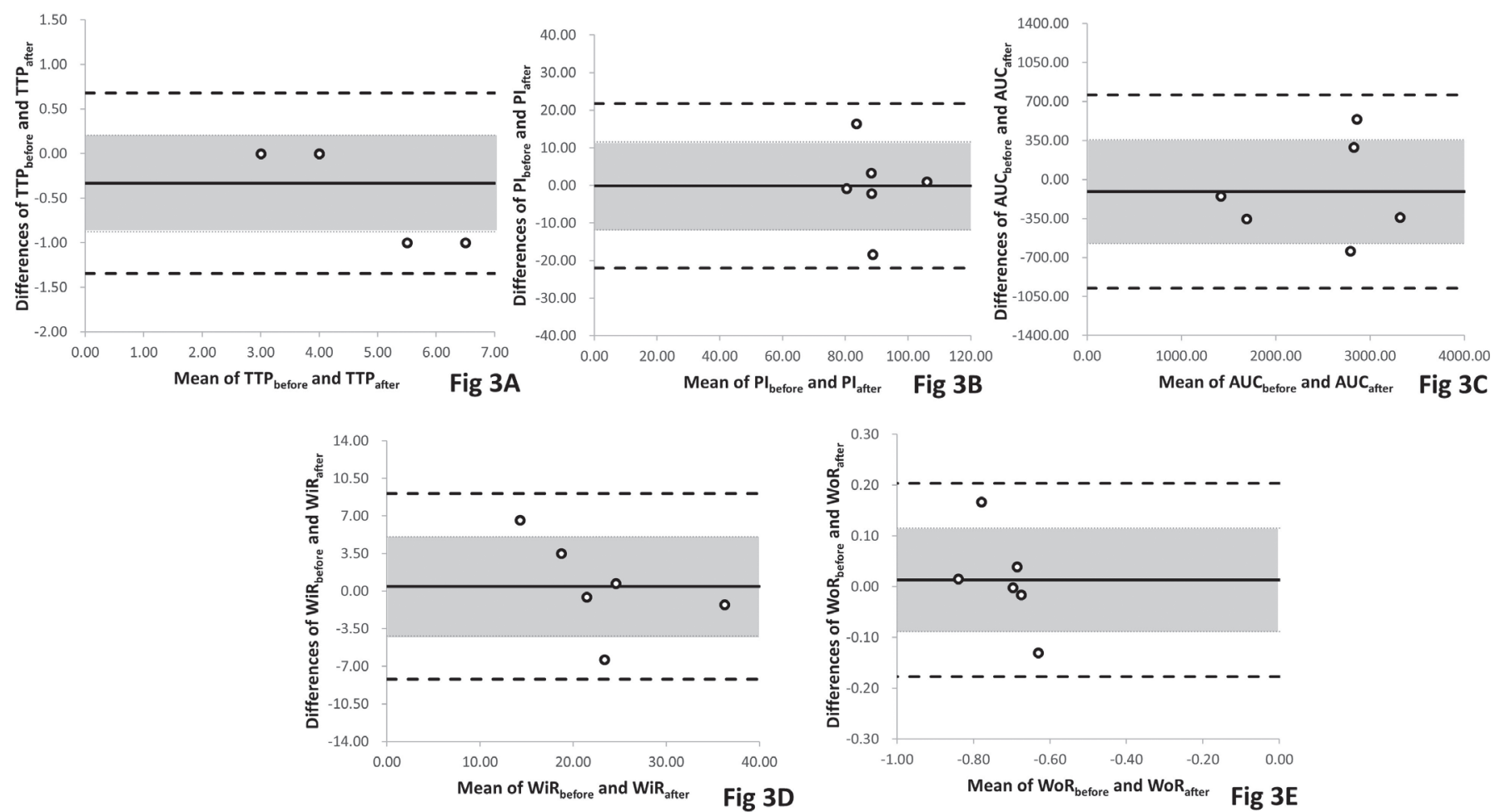

Fig. 3. Bland-Altman plots of the differences among perfusion parameters before and after sedation with mean differences [(bias), continuous line], $95 \%$ confidence interval of mean difference (shaded areas), and limit of agreement (dashed line). Bias (95\% CI) between TTP before and TTP after was -0.33 ( -0.88 to 0.21$)$ [A]. Bias $(95 \% \mathrm{CI})$ between $\mathrm{PI}_{\text {before }}$ and $\mathrm{PI}_{\text {after }}$ was -0.12 (-11.84 to 11.56) [B]. Bias $(95 \% \mathrm{CI})$ between $\mathrm{AUC}$ before and $\mathrm{AUC}_{\mathrm{after}}$ was $-108.07(-572.53$ to 356.40$)[\mathrm{C}]$. Bias $(95 \% \mathrm{CI})$ between $\mathrm{WiR}_{\text {before }}$ and $\mathrm{WiR}_{\text {after }}$ was $0.44(-4.20$ to 5.07$)[\mathrm{D}] . \mathrm{Bias}(95 \% \mathrm{CI})$ between $W_{0} R_{\text {before }}$ and $\mathrm{WoR}_{\text {after }}$ was $0.01(-0.09$ to 0.12$)[\mathrm{E}]$.

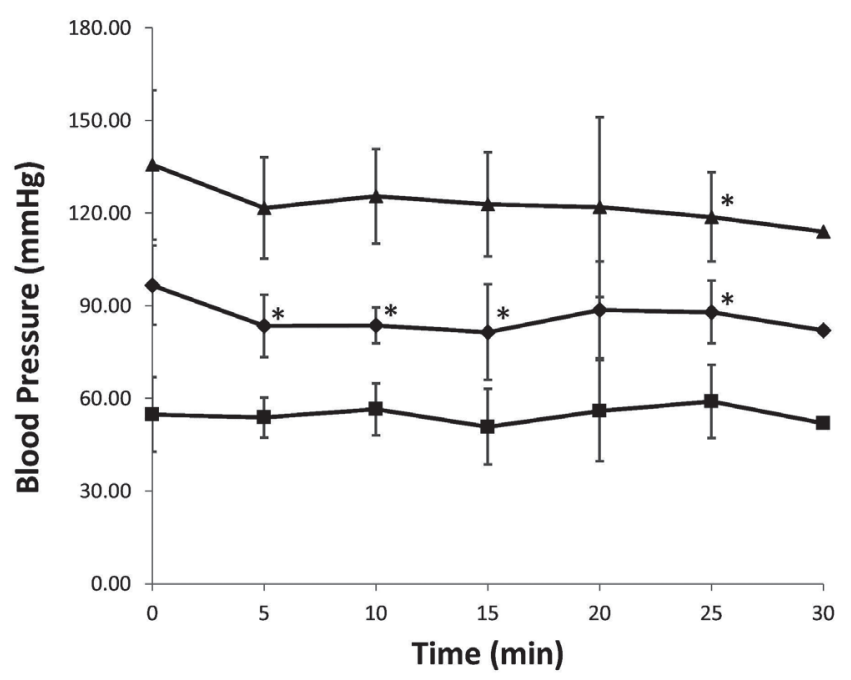

$\leftarrow$ SAP $\rightarrow$-DAP $\rightarrow$ MAP

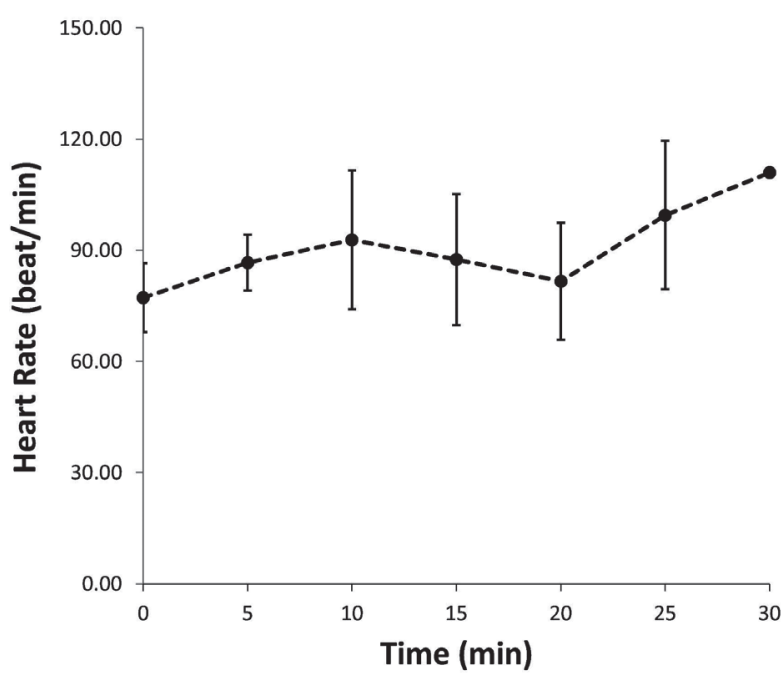

Fig 4A
Fig 4B

Fig. 4. Blood pressure (systolic, diastolic, mean arterial pressure=SAP, DAP, MAP, respectively) [A] and heart rate (HR) [B] following the sedative administration of a combination of butorphanol and midazolam. The SAP is significantly lower at 25 min, while the MAP is significantly lower at 5, 10, 15 and 25 min when compared to baseline (time $=0$, before sedative administration). DAP and HR do not show significant difference at any time point. 
butorphanol [15].

Autoregulation of intestinal blood supply might play a role to maintain duodenal perfusion, despite the reduction of systemic blood pressure which more likely caused by midazolam as it depresses the sympathetic nerve activity and causes a decrease in systemic vascular resistance $[12,13]$. The autoregulation occurs to preserve nutrient and oxygen supply toward the absorption sites [9]. In our study, ROI was placed in the mucosa which was found to have more potent local circulatory control mechanism than muscular layer [29]. A previous study reported that blood flow and blood volume remain unchanged in the intestinal mucosa of hypovolemic dogs, even though a decrease in the superior mesenteric artery (SMA) flow was detected together with an altered cardiac output and mean arterial pressure [3]. Reduced blood flow towards splanchnic organs including intestine was suspected in dogs after administration of midazolam as a single anesthetic agent [7], whereas butorphanol was suggested to cause a decrease in the intestinal blood flow of halothane-anesthetized ponies [30]. There was neither previous report explaining how butorphanol-midazolam in a sedative dosage affect canine splanchnic blood flow nor the blood distribution within intestinal wall, but considering the result of the previous studies, butorphanol-midazolam might cause changes in the SMA flow that induced autoregulation in the duodenum of our dogs. Apart from these mechanisms, very small changes of duodenal perfusion which could only be detected by a highly accurate technique such as radiolabel tracking might only cause a negligible effect on the CEUSderived perfusion parameters.

This study has a number of limitations. First, this study only evaluated the combination of butorphanol and midazolam. The sedative or anesthetic agent which have a large influence on cardiovascular system was not examined as a positive control, so the effect of cardiovascular change induced by such agents on CEUS-derived duodenal perfusion parameters is unknown. In a previous study, dexmedetomidine was reported to cause prolonged arrival time and time to peak intensity, as well as a lower wash-in rate on CEUS evaluation of canine small intestine [27]. Dexmedetomidine causes peripheral vasoconstriction resulting in transitory hypertension and bradycardia at the initial phase after administration and followed by lower cardiac output and reduction in perfusion of peripheral organs [17]. Second, healthy and relatively young dogs were subjected in our study. In clinical practice, however, dogs with chronic intestinal diseases such as idiopathic inflammatory bowel disease and intestinal lymphoma are mostly middle-aged dogs $[1,4,6]$. Butorphanol-midazolam was reported to cause mild to moderate effect in healthy and young dogs but may cause a deeper effect in sick and old animals [14,23]. Therefore, this combination must be used with caution in a clinical setting. Moreover, the inter-sonographer variability was not examined in the current study.

From the results of current study, it can be concluded that a combination of butorphanol and midazolam did not cause a significant change in duodenal CEUS-derived perfusion parameters of healthy dogs. This finding indicated that the combination could be a good option for sedation prior to duodenal CEUS in debilitated dogs.

\section{REFERENCES}

1. Allenspach, K., Wieland, B., Gröne, A. and Gaschen, F. 2007. Chronic enteropathies in dogs: evaluation of risk factors for negative outcome. J. Vet. Intern. Med. 21: 700-708. [Medline] [CrossRef]

2. Bland, J. M. and Altman, D. G. 1999. Measuring agreement in method comparison studies. Stat. Methods Med. Res. 8: 135-160. [Medline] [CrossRef]

3. Connolly, H. V., Maginniss, L. A. and Schumacker, P. T. 1997. Transit time heterogeneity in canine small intestine: significance for oxygen transport. J. Clin. Invest. 99: 228-238. [Medline] [CrossRef]

4. Craven, M., Simpson, J. W., Ridyard, A. E. and Chandler, M. L. 2004. Canine inflammatory bowel disease: retrospective analysis of diagnosis and outcome in 80 cases (1995-2002). J. Small Anim. Pract. 45: 336-342. [Medline] [CrossRef]

5. Dietrich, C. F., Averkiou, M. A., Correas, J. M., Lassau, N., Leen, E. and Piscaglia, F. 2012. An EFSUMB introduction into Dynamic ContrastEnhanced Ultrasound (DCE-US) for quantification of tumour perfusion. Ultraschall Med. 33: 344-351. [Medline] [CrossRef]

6. Frank, J. D., Reimer, S. B., Kass, P. H. and Kiupel, M. 2007. Clinical outcomes of 30 cases (1997-2004) of canine gastrointestinal lymphoma. J. Am. Anim. Hosp. Assoc. 43: 313-321. [Medline] [CrossRef]

7. Gelman, S., Reves, J. G. and Harris, D. 1983. Circulatory responses to midazolam anesthesia: emphasis on canine splanchnic circulation. Anesth. Analg. 62: 135-139. [Medline] [CrossRef]

8. Girlich, C., Schacherer, D., Jung, E. M., Schreyer, A. and Büttner, R. 2012. Comparison between a clinical activity index (Harvey-Bradshaw-Index), laboratory inflammation markers and quantitative assessment of bowel wall vascularization by contrast-enhanced ultrasound in Crohn's disease. Eur. J. Radiol. 81: 1105-1109. [Medline] [CrossRef]

9. Granger, D. N., Richardson, P. D. I., Kvietys, P. R. and Mortillaro, N. A. 1980. Intestinal blood flow. Gastroenterology 78: 837-863. [Medline]

10. Jiménez, D. A., O’Brien, R. T., Wallace, J. D. and Klocke, E. 2011. Intraoperative contrast-enhanced ultrasonography of normal canine jejunum. Vet. Radiol. Ultrasound 52: 196-200. [Medline] [CrossRef]

11. Johnson-Neitman, J. L., O'Brien, R. T. and Wallace, J. D. 2012. Quantitative perfusion analysis of the pancreas and duodenum in healthy dogs by use of contrast-enhanced ultrasonography. Am. J. Vet. Res. 73: 385-392. [Medline] [CrossRef]

12. Jones, D. J., Stehling, L. C. and Zauder, H. L. 1979. Cardiovascular responses to diazepam and midazolam maleate in the dog. Anesthesiology 51: 430-434. [Medline] [CrossRef]

13. Kobayashi, Y., Muldoon, S. M., Kiyose, M., Hagiwara, T., Kumasaka, S. and Okabe, E. 1998. Inhibition by midazolam of the adrenergic function in the isolated canine mesenteric vein. Acta Anaesthesiol. Scand. 42: 1157-1163. [Medline] [CrossRef]

14. Kojima, K., Nishimura, R., Mutoh, T., Takao, K., Matsunaga, S., Mochizuki, M. and Sasaki, N. 1999. Comparison of cardiopulmonary effects of medetomidine-midazolam, acepromazine-butorphanol and midazolam-butorphanol in dogs. Zentralbl. Veterinarmed. A 46: 353-359. [Medline] [CrossRef]

15. Kojima, K., Nishimura, R., Mutoh, T., Takao, K., Matsunaga, S., Mochizuki, M. and Sasaki, N. 1999. Comparison of sedative effects of medetomidine-midazolam, acepromazine-butorphanol and midazolam-butorphanol in dogs. Zentralbl. Veterinarmed. A 46: 141-148. [Medline] [CrossRef] 
16. Lamont, L. A. and Mathews, K. A. 2007. Opioid, nonsteroidal anti-inflammatories, and analgesic adjuvants. pp. 241-271. In: Lumb\&Jones Veterinary Anesthesia and Analgesia, 4th ed. (Tranquilli, W. J., Thurmon, J. C. and Grimm, K. A. eds.), Blackwell, Oxford.

17. Lawrence, C. J., Prinzen, F. W. and de Lange, S. 1996. The effect of dexmedetomidine on nutrient organ blood flow. Anesth. Analg. 83: 1160-1165. [Medline] [CrossRef]

18. Lemke, K. A. 2007. Anticholinergic and sedatives. pp. 203-239. In: Lumb\&Jones Veterinary Anesthesia and Analgesia, 4th ed. (Tranquilli, W. J., Thurmon, J. C. and Grimm, K. A. eds.), Blackwell, Oxford.

19. Lim, S. Y., Nakamura, K., Morishita, K., Sasaki, N., Murakami, M., Osuga, T., Ohta, H., Yamasaki, M. and Takiguchi, M. 2013. Qualitative and quantitative contrast enhanced ultrasonography of the pancreas using bolus injection and continuous infusion methods in normal dogs. J. Vet. Med. Sci. 75: 1601-1607. [Medline] [CrossRef]

20. Medellin-Kowalewski, A., Wilkens, R., Wilson, A., Ruan, J. and Wilson, S. R. 2016. Quantitative contrast-enhanced ultrasound parameters in Crohn disease: their role in disease activity determination with ultrasound. AJR Am. J. Roentgenol. 206: 64-73. [Medline] [CrossRef]

21. Nakamura, K., Sasaki, N., Yoshikawa, M., Ohta, H., Hwang, S. J., Mimura, T., Yamasaki, M. and Takiguchi, M. 2009. Quantitative contrastenhanced ultrasonography of canine spleen. Vet. Radiol. Ultrasound 50: 104-108. [Medline] [CrossRef]

22. Nisa, K., Lim, S. Y., Shinohara, M., Nagata, N., Sasaoka, K., Dermlim, A., Leela-Arporn, R., Morita, T., Yokoyama, N., Osuga, T., Sasaki, N., Morishita, K., Nakamura, K., Ohta, H. and Takiguchi, M. 2017. Repeatability and reproducibility of quantitative contrast-enhanced ultrasonography for assessing duodenal perfusion in healthy dogs. J. Vet. Med. Sci. 79: 1585-1590 [Medline] [CrossRef].

23. Novellas, R., Ruiz de Gopegui, R. and Espada, Y. 2007. Effects of sedation with midazolam and butorphanol on resistive and pulsatility indices in healthy dogs. Vet. Radiol. Ultrasound 48: 276-280. [Medline] [CrossRef]

24. Nylund, K., Jirik, R., Mezl, M., Leh, S., Hausken, T., Pfeffer, F., Ødegaard, S., Taxt, T. and Gilja, O. H. 2013. Quantitative contrast-enhanced ultrasound comparison between inflammatory and fibrotic lesions in patients with Crohn's disease. Ultrasound Med. Biol. 39: 1197-1206. [Medline] [CrossRef]

25. Quaia, E., Sozzi, M., Angileri, R., Gennari, A. G. and Cova, M. A. 2016. Time-intensity curves obtained after microbubble injection can be used to differentiate responders from nonresponders among patients with clinically active Crohn disease after 6 weeks of pharmacologic treatment. Radiology 281: 606-616. [Medline] [CrossRef]

26. Quaia, E., De Paoli, L., Stocca, T., Cabibbo, B., Casagrande, F. and Cova, M. A. 2012. The value of small bowel wall contrast enhancement after sulfur hexafluoride-filled microbubble injection to differentiate inflammatory from fibrotic strictures in patients with Crohn's disease. Ultrasound Med. Biol. 38: 1324-1332. [Medline] [CrossRef]

27. Restitutti, F., Laitinen, M. R., Raekallio, M. R., Vainionpää, M., O’Brien, R. T., Kuusela, E. and Vainio, O. M. 2013. Effect of MK-467 on organ blood flow parameters detected by contrast-enhanced ultrasound in dogs treated with dexmedetomidine. Vet. Anaesth. Analg. 40: e48-e56. [Medline] [CrossRef]

28. Rossi, F., Fina, C., Stock, E., Vanderperren, K., Duchateau, L. and Saunders, J. H. 2016. Effect of sedation on contrast-enhanced ultrasonography of the spleen in healthy dogs. Vet. Radiol. Ultrasound 57: 276-281. [Medline] [CrossRef]

29. Shepherd, A. P. and Riedel, G. L. 1988. Intramural distribution of intestinal blood flow during sympathetic stimulation. Am. J. Physiol. 255: H1091H1095. [Medline]

30. Stick, J. A., Loeffler, B. S., Arden, W. A. and Chou, C. C. 1989. Effects of butorphanol tartrate on arterial pressure, jejunal blood flow, vascular resistance, $\mathrm{O} 2$ extraction, and $\mathrm{O} 2$ uptake in halothane-anesthetized ponies. Am. J. Vet. Res. 50: 1202-1206. [Medline]

31. Stock, E., Vanderperren, K., Van der Vekens, E., Haers, H., Duchateau, L., Polis, I., Hesta, M. and Saunders, J. H. 2014. The effect of anesthesia with propofol and sedation with butorphanol on quantitative contrast-enhanced ultrasonography of the healthy feline kidney. Vet. J. 202: 637-639. [Medline] [CrossRef]

32. Tang, M. X., Mulvana, H., Gauthier, T., Lim, A. K. P., Cosgrove, D. O., Eckersley, R. J. and Stride, E. 2011. Quantitative contrast-enhanced ultrasound imaging: a review of sources of variability. Interface Focus 1: 520-539. [Medline] [CrossRef]

33. Waller, K. R., O'Brien, R. T. and Zagzebski, J. A. 2007. Quantitative contrast ultrasound analysis of renal perfusion in normal dogs. Vet. Radiol. Ultrasound 48: 373-377. [Medline] [CrossRef]

34. Ziegler, L. E., O’Brien, R. T., Waller, K. R. and Zagzebski, J. A. 2003. Quantitative contrast harmonic ultrasound imaging of normal canine liver. Vet. Radiol. Ultrasound 44: 451-454. [Medline] [CrossRef] 